\title{
Livestock grazing-induced large-scale biotic homogenization in arid Mediterranean steppe rangelands
}

\author{
Merdas Saifi ${ }^{1}$, Yacine Kouba ${ }^{2}$, Tewfik Mostephaoui ${ }^{1}$, Yassine Farhi ${ }^{1}$, and Haroun \\ Chenchouni ${ }^{2,3}$ \\ ${ }^{1}$ Centre of scientific and technical research on arid regions \\ ${ }^{2}$ University of Oum-El-Bouaghi \\ ${ }^{3}$ University of Tebessa
}

July 14, 2021

\begin{abstract}
Despite many studies explored the effect of livestock grazing on plant communities, the response of species composition and diversity to livestock grazing in arid rangelands remain ambiguous. This study examined the effects of livestock grazing on plant communities in arid steppe rangelands of North Africa. Plant diversity of annual species, perennial species and all species combined was measured and compared between grazed and grazing-excluded areas. We also examined the relative importance of species turnover and community nestedness. Moreover, the effects of livestock grazing on beta diversity at local among transects and landscape among sites scales were examined using the multiplicative diversity partitioning. Results revealed that livestock grazing significantly decreased the alpha diversity of all species combined and the diversity of annual plants. Livestock grazing induced a shift in plant community composition where most of species composition variation ( $\sim 74 \%)$ was due to infrequent species replacement 'turnover' between the two management types rather than nestedness ( $\sim 26 \%)$. Results revealed also that among transects, beta diversity was higher in grazed steppes than in grazing-excluded steppes. Whereas, among sites, beta diversity was lower in grazed steppes compared to grazing-excluded steppes. These findings suggest that livestock grazing in arid steppe rangelands increases the variation in plant species composition at a local spatial scale and engenders vegetation homogeneity at landscape spatial scale. Therefore, the implementation of appropriate management practices such as short-term grazing exclusion is mandatory to prevent these ecosystems from large scale biotic homogenization.
\end{abstract}

\section{Hosted file}

Maintext 2021-07-11.pdf available at https://authorea.com/users/399712/articles/512173livestock-grazing-induced-large-scale-biotic-homogenization-in-arid-mediterraneansteppe-rangelands 


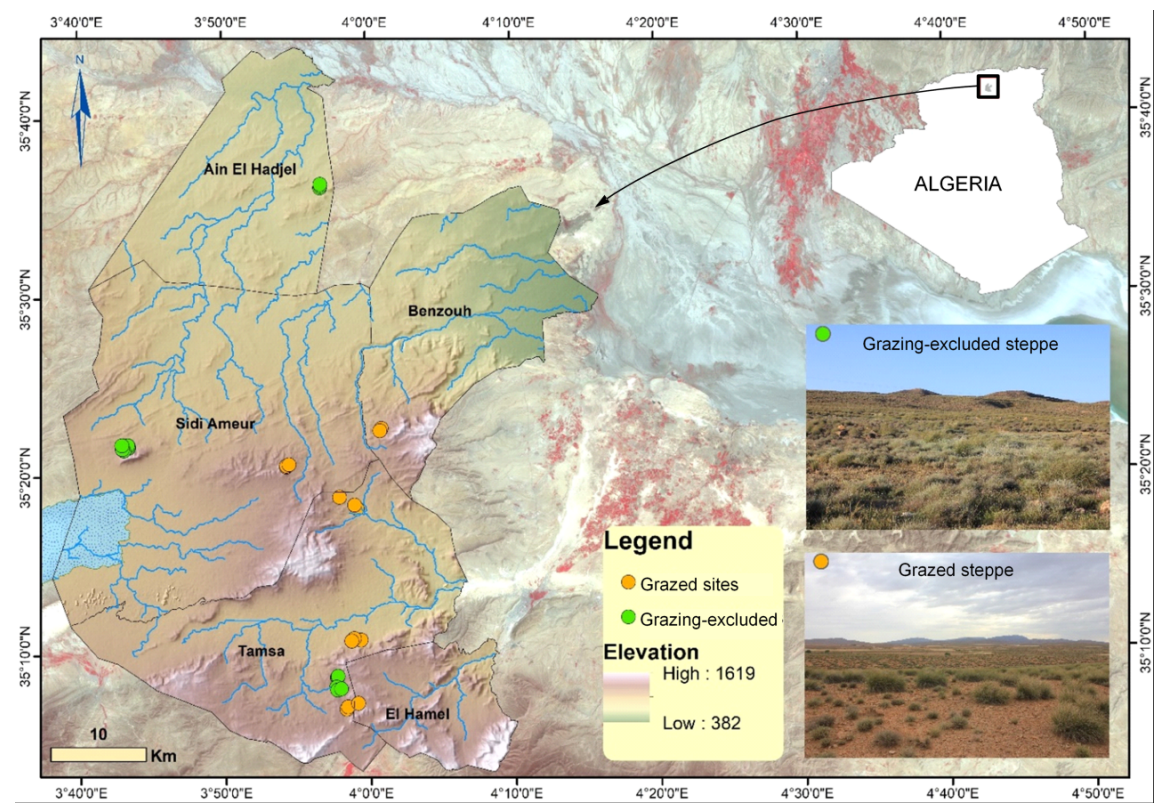

FIGURE 1. Location of the study area in the central steppe rangelands of Algeria. Orange solid points $(*)$ indicate locations of sampled sites in grazed steppes and green solid points $\left(^{*}\right)$ represent grazing-excluded sites 

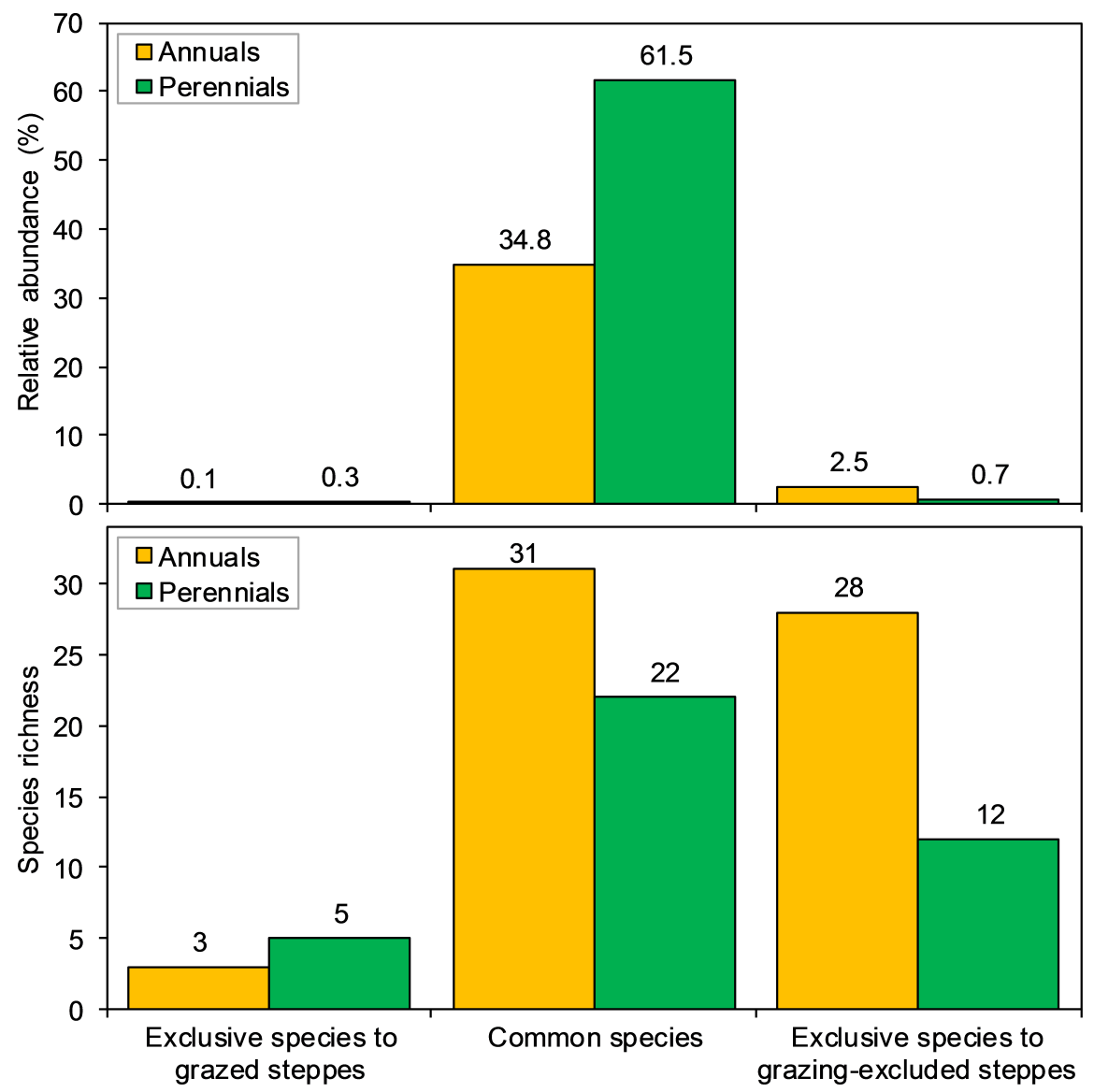

FIGURE 2. Plant relative abundance (in\%) and species richness for perennials and annuals in grazed and grazing-excluded steppe rangelands of arid lands of Algeria. 


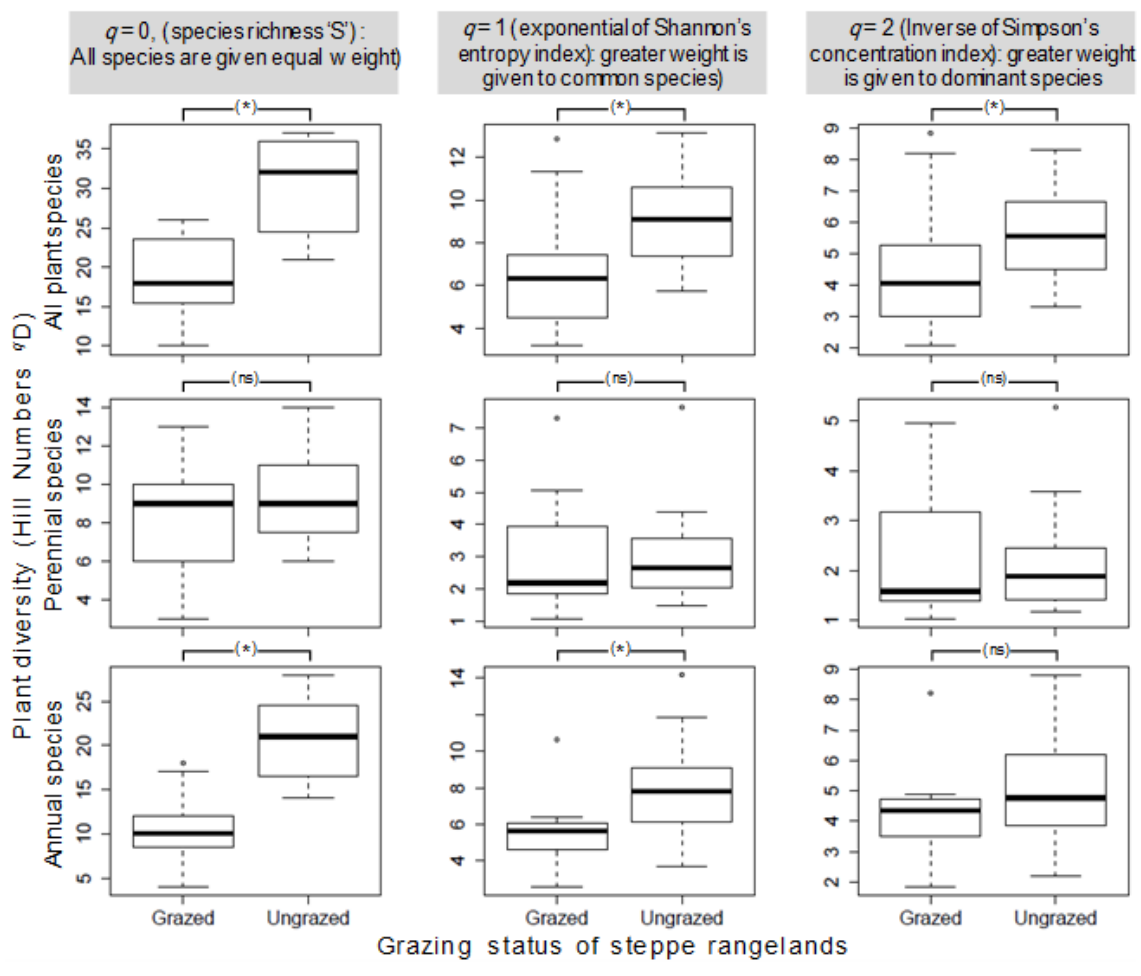

FIGURE 3. Comparing alpha diversities for all plant species combined, perennials and annuals, between grazed and grazing-excluded "ungrazed" areas of the steppe rangelands of Algeria. Results of GLMMs tests are included in the figure as an asterisk $\left(^{*}\right)$ indicating significant differences $(P>0.05)$, and (ns) no significant differences

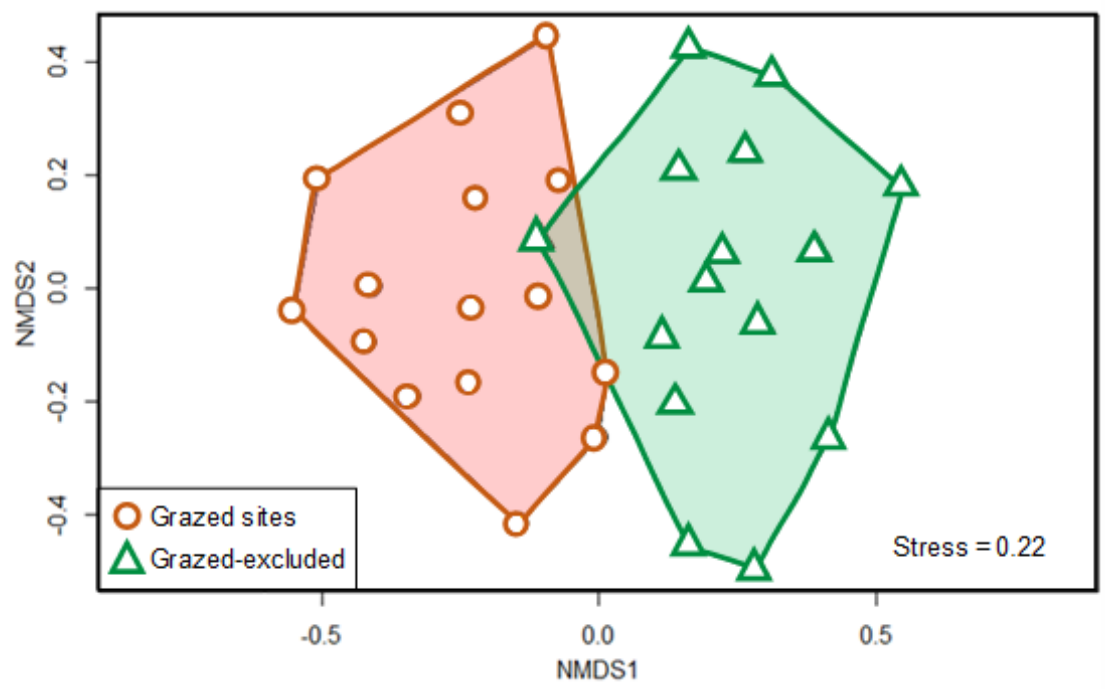

FIGURE 4. Non-metrical multidimensional scaling (NMDS) ordination based on Bray-Curtis similarities using 4th-root transformation. Each symbol-point represents a $200-\mathrm{m}$ transect used in plant sampling. 


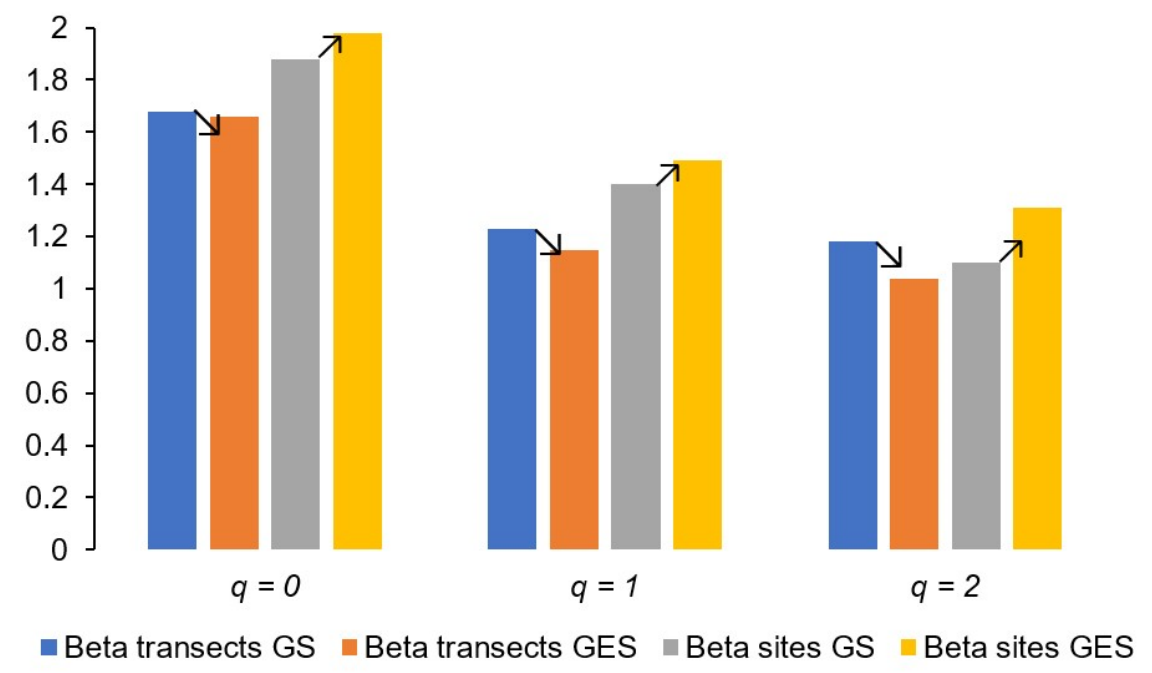

Figure 1: This is a caption

FIGURE 5. Comparing beta diversities between grazed and grazing-excluded in the arid steppes rangelands of Algeria. Diversity was quantified using the Hill numbers, with $q=0$ (all species are given equal weight), $q=1$ (greater weight is given to common species), or $q=2$ (greater weight is given to dominant species). "Beta transects GS" and "Beta transects GES" refer to among transects beta diversity (grain = transect and extent = site) in grazed and grazing-excluded steppes, respectively; "Beta sites GS" and "Beta sites GES" refer to among sites beta diversity (grain $=$ site and extent $=$ management type) in grazed and grazing-excluded steppes, respectively. 